Supplement of Primate Biol., 6, 7-16, 2019

https://doi.org/10.5194/pb-6-7-2019-supplement

Primate

(c) Author(s) 2019. This work is distributed under

the Creative Commons Attribution 4.0 License.

(c) (1)

Biology

Supplement of

\title{
Is Colobus guereza gallarum a valid endemic Ethiopian taxon?
}

Dietmar Zinner et al.

Correspondence to: Dietmar Zinner (dzinner@dpz.eu)

The copyright of individual parts of the supplement might differ from the CC BY 4.0 License. 
Supplement Material

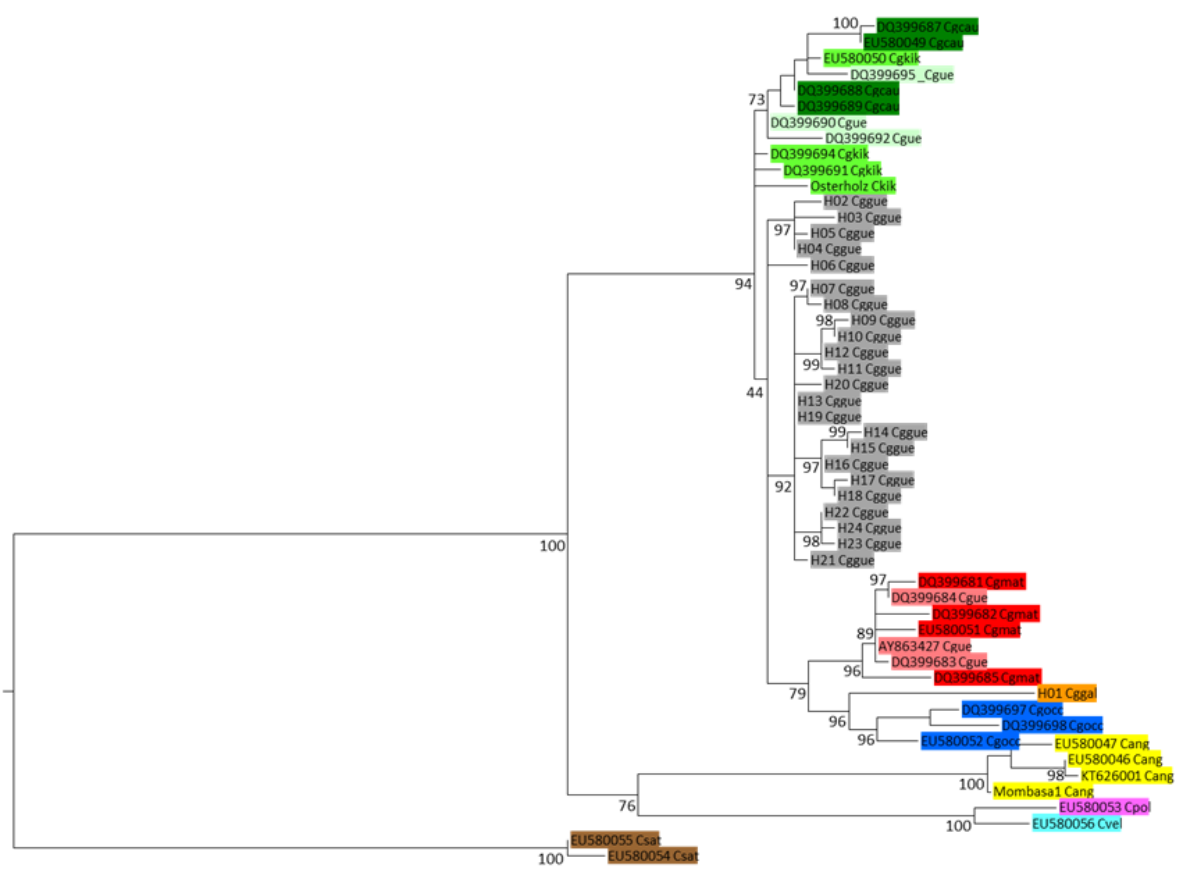

Figure S1a. ML tree $(\mathrm{NADH})$. Csat $=$ Colobus satanas, Cvel $=$ C. vellerosus, $\mathrm{Cpol}=C$. polykomos, Cang $=$ C. angolensis, Cgocc $=C$. guereza occidentalis, Cggal $=C$. g. gallarum, Cgmat $=$ C. g. matschiei, Cggue $=$ C. g. guereza, Cgkik $=$ C. g. kikuyuensis, Cgcau $=$ C. $g$. caudatus, Cgue $=C$. guereza ssp.. Node labels refer to ML BS values.

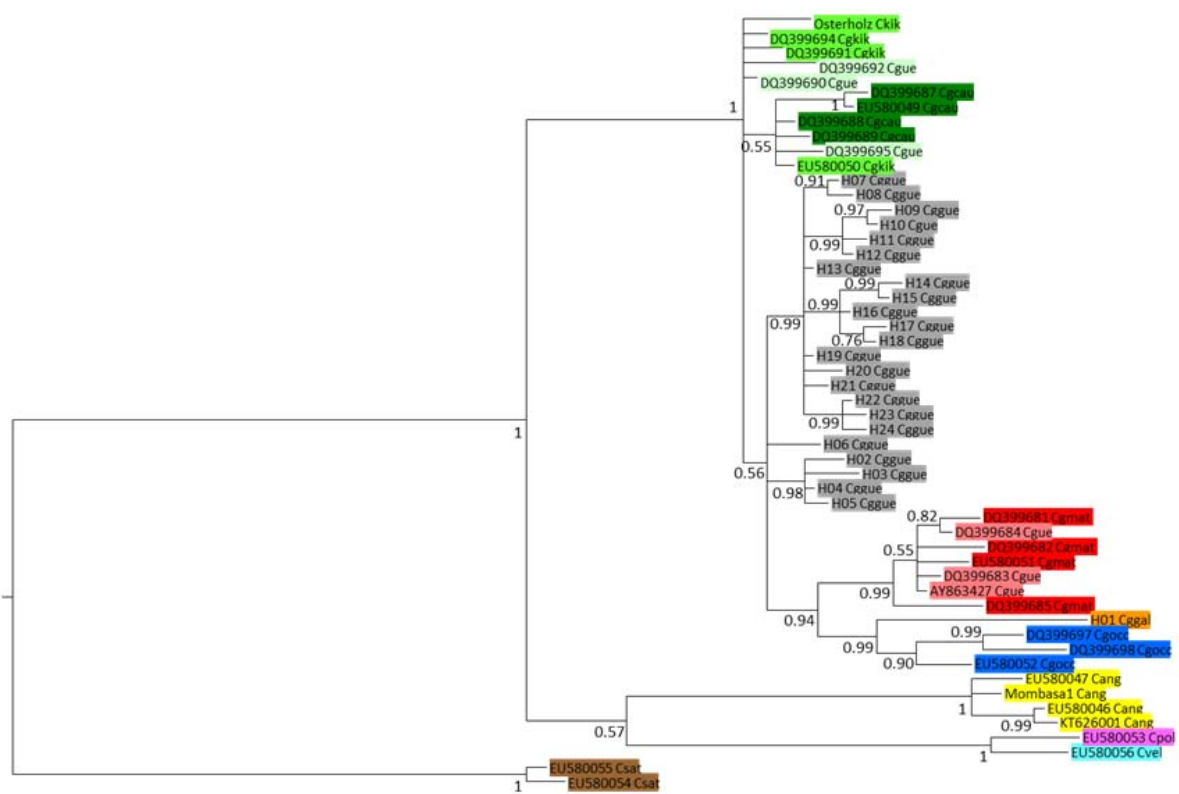

Figure S1b. Bayesian tree $(\mathrm{NADH})$. Csat $=$ Colobus satanas, Cvel $=$ C. vellerosus, $\mathrm{Cpol}=C$. polykomos, Cang $=C$. angolensis, Cgocc $=C$. guereza occidentalis, Cggal $=C$. g. gallarum, Cgmat $=$ C. g. matschiei, Cggue $=$ C. g. guereza, Cgkik $=$ C. g. kikuyuensis, Cgcau $=$ C. $g$. caudatus, Cgue $=$ C. guereza ssp.. Node labels refer to Bayesian PPs. 


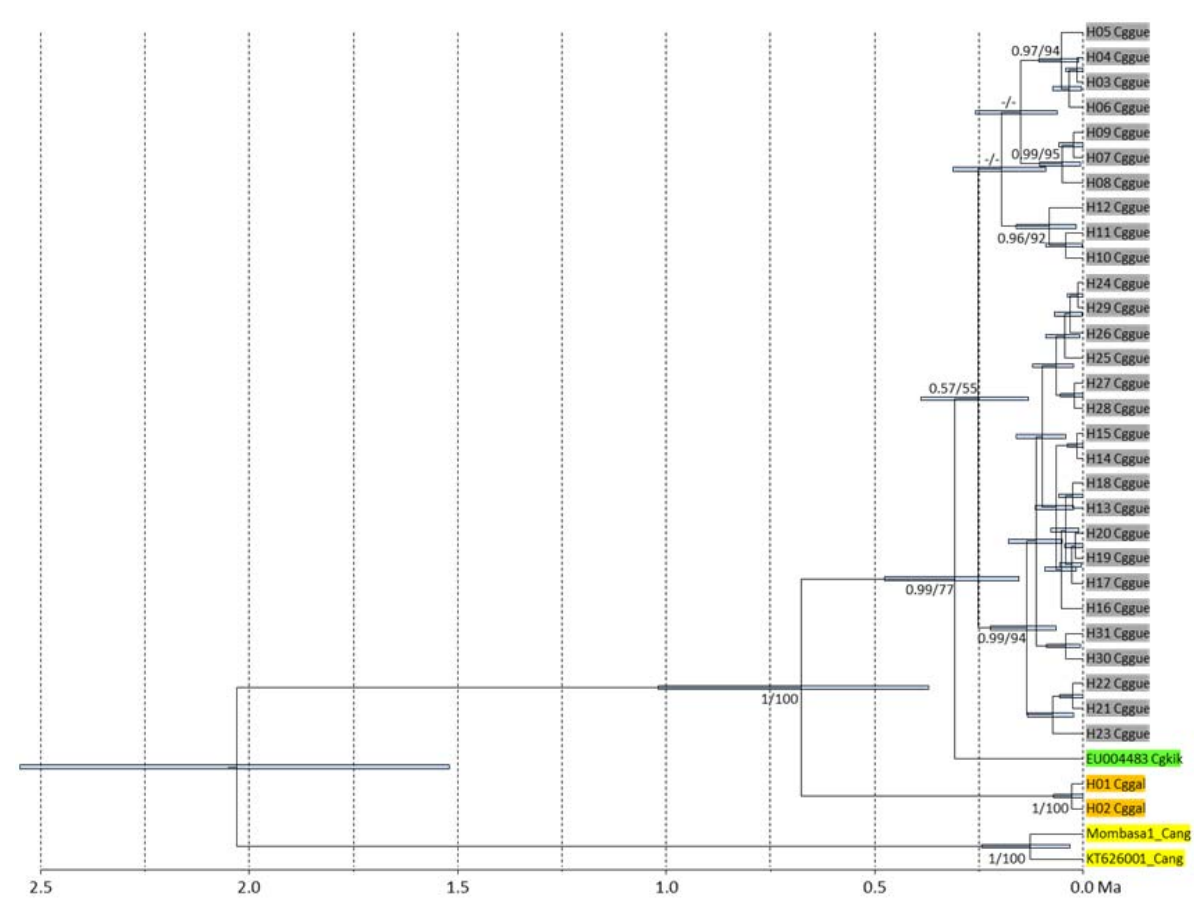

Figure S2a. Ultrametric tree showing phylogenetic relationships and divergence times of Colobus mtDNA lineages (based on 1140 bp of cytb). Tip labels refer to Colobus haplotypes (see Table S1). Cang = C. angolensis, Cggal = C. g. gallarum, Cggue = C. g. guereza, Cgkik = C. g. kikuyuensis. Node labels refer to ML BS and Bayesian PP values. The time scale below the tree indicates million years ago.

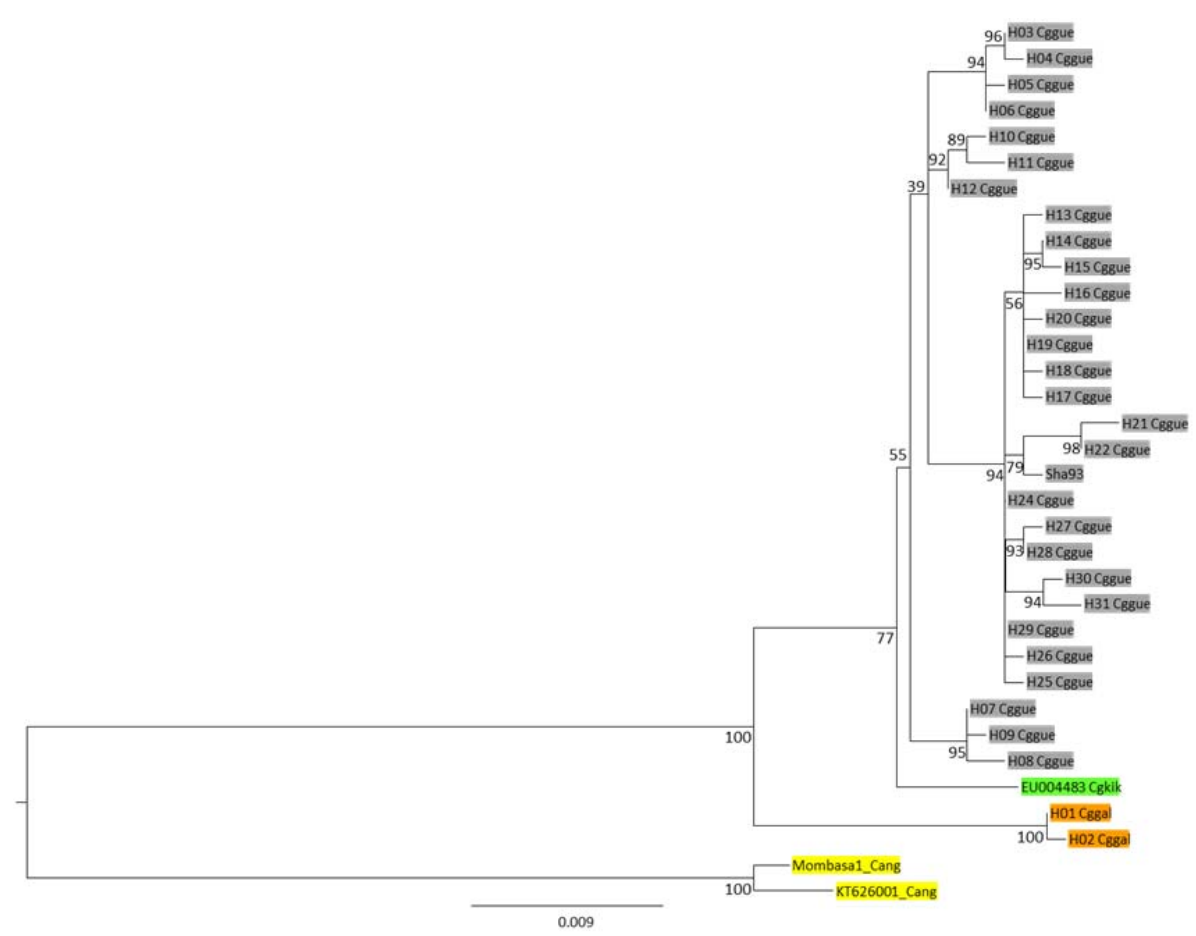

Figure S2b. ML tree (cytb). Csat $=$ Colobus satanas, Cvel $=C$. vellerosus, $\mathrm{Cpol}=C$. polykomos, Cang $=C$. angolensis, Cgocc $=C$. guereza occidentalis, Cggal $=C . g$. gallarum, Cgmat $=$ C. g. matschiei, Cggue $=$ C.g. guereza, Cgkik $=$ C. g. kikuyuensis, Cgcau $=$ C. $g$. caudatus, Cgue $=$ C. guereza ssp.. Node labels refer to ML BS values. 


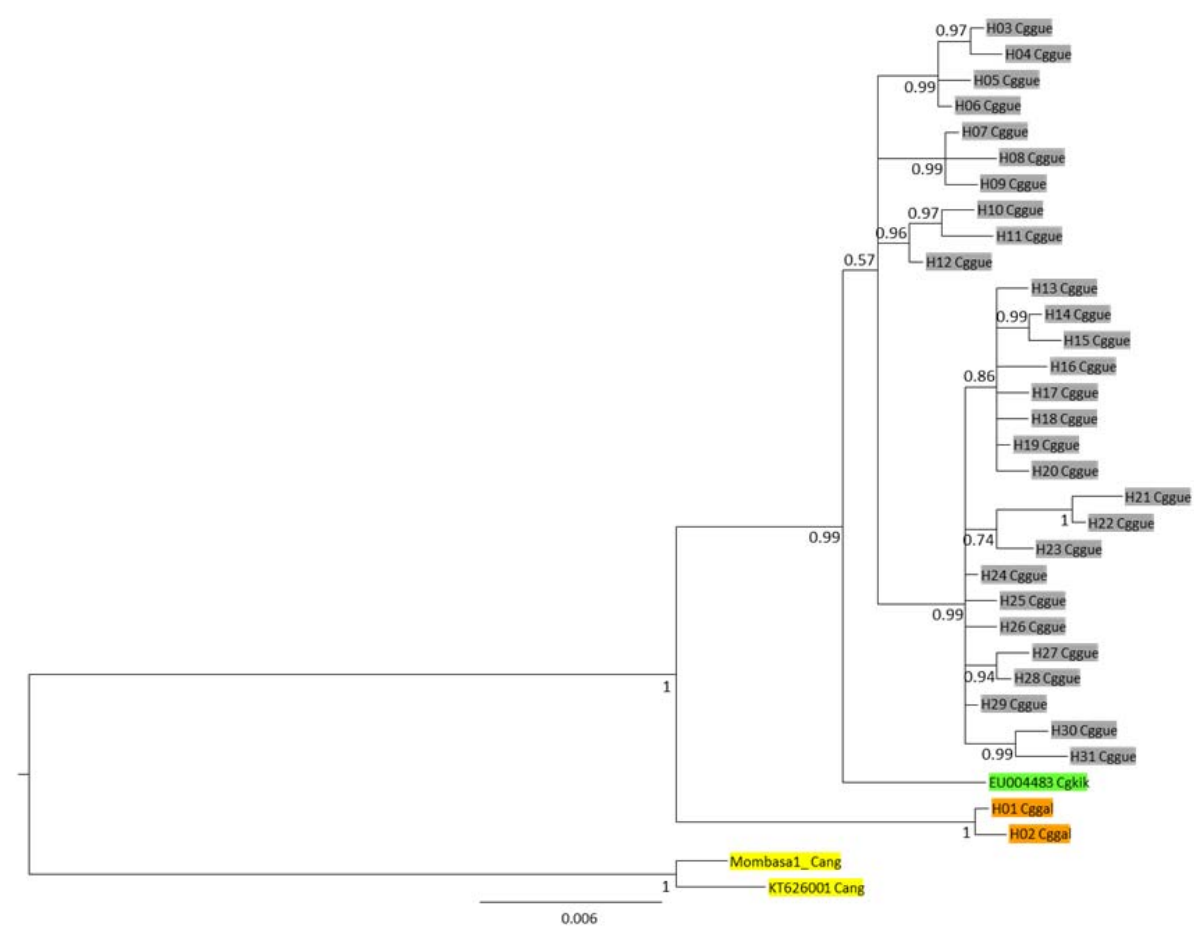

Figure S2c. Bayesian tree (cytb). Csat $=$ Colobus satanas, Cvel $=C$. vellerosus, $\mathrm{Cpol}=C$. polykomos, Cang $=C$. angolensis, Cgocc $=C$. guereza occidentalis, Cggal =C. g. gallarum, Cgmat $=$ C. g. matschiei, Cggue $=$ C. g. guereza, Cgkik $=$ C. g. kikuyuensis, Cgcau $=$ C. $g$. caudatus, Cgue $=$ C. guereza ssp.. Node labels refer to Bayesian PPs. 


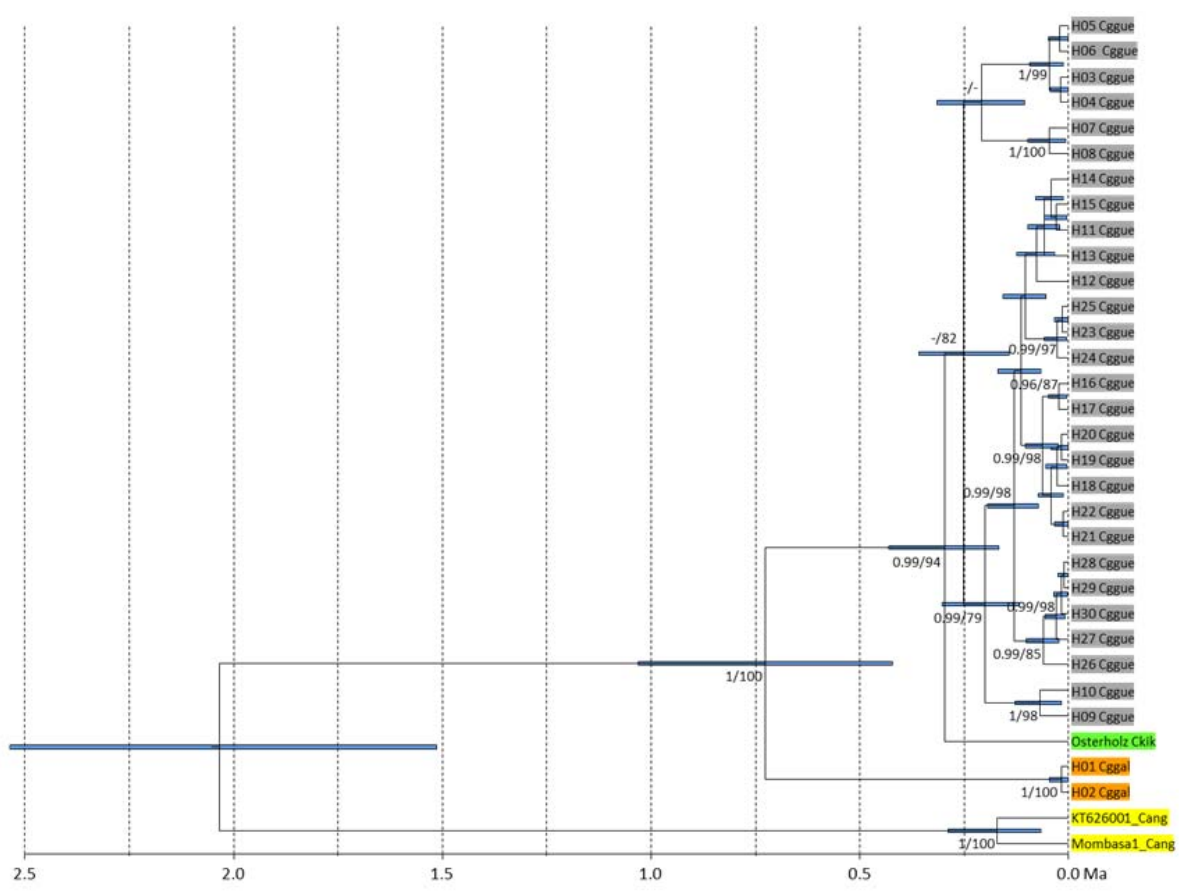

Figure S3a. Ultrametric tree showing phylogenetic relationships and divergence times of Colobus mtDNA lineages (based on concatenated 1930 bp of NADH and cytb). Tip labels refer to Colobus haplotypes (see Table S1). Cang $=$ C. angolensis, Cggal $=C$. g. gallarum, Cggue $=$ C. g. guereza, Cgkik $=$ C. g. kikuyuensis. Node labels refer to ML BS and Bayesian PP values. The time scale below the tree indicates million years ago.

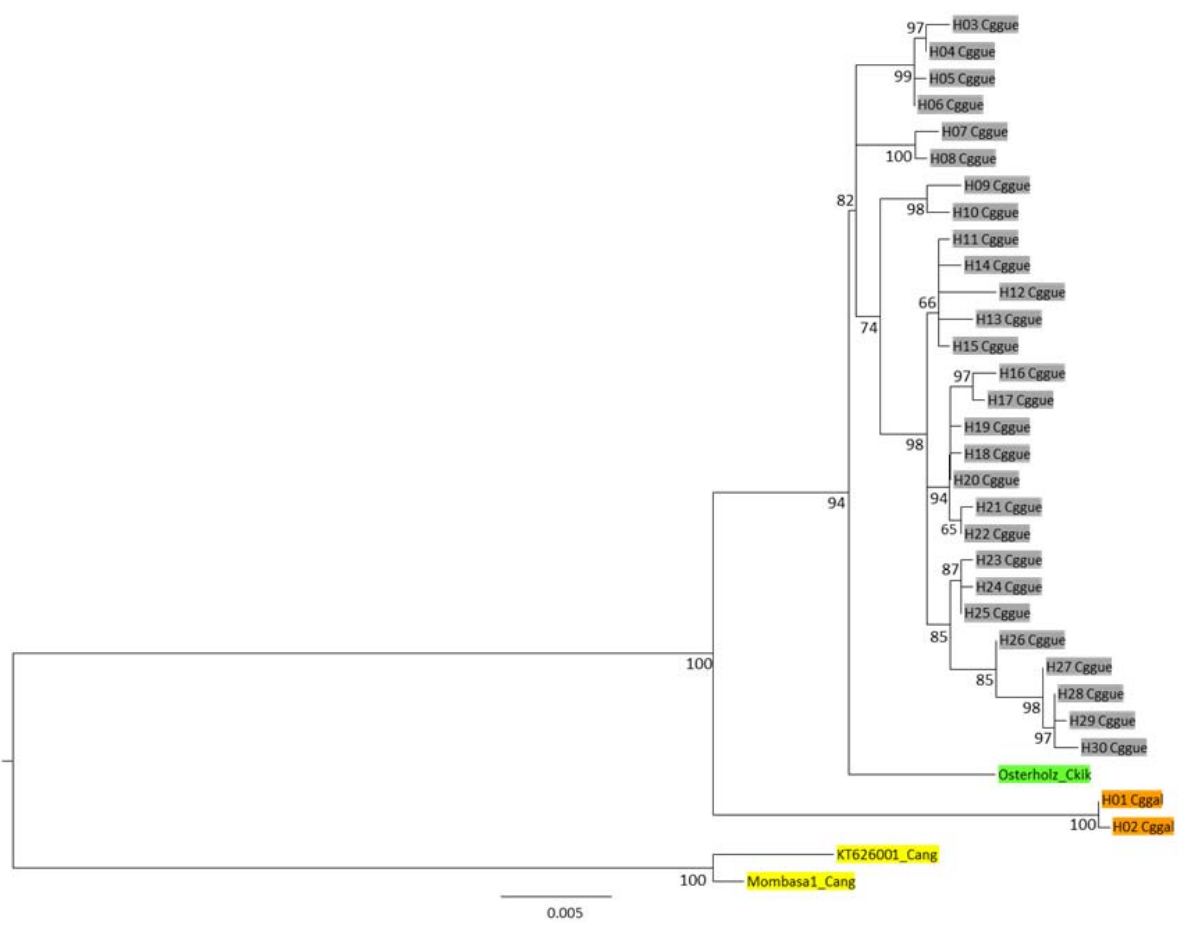

Figure S3b. ML tree (concatenated). Csat $=$ Colobus satanas, Cvel $=$ C. vellerosus, Cpol $=C$. polykomos, Cang $=$ C. angolensis, Cgocc $=$ C. guereza occidentalis, Cggal $=$ C. g. gallarum, Cgmat $=$ C. g. matschiei, Cggue $=$ C.g. guereza, Cgkik $=$ C. g. kikuyuensis, Cgcau $=$ C. $g$. caudatus, Cgue $=C$. guereza ssp.. Node labels refer to ML BS values. 


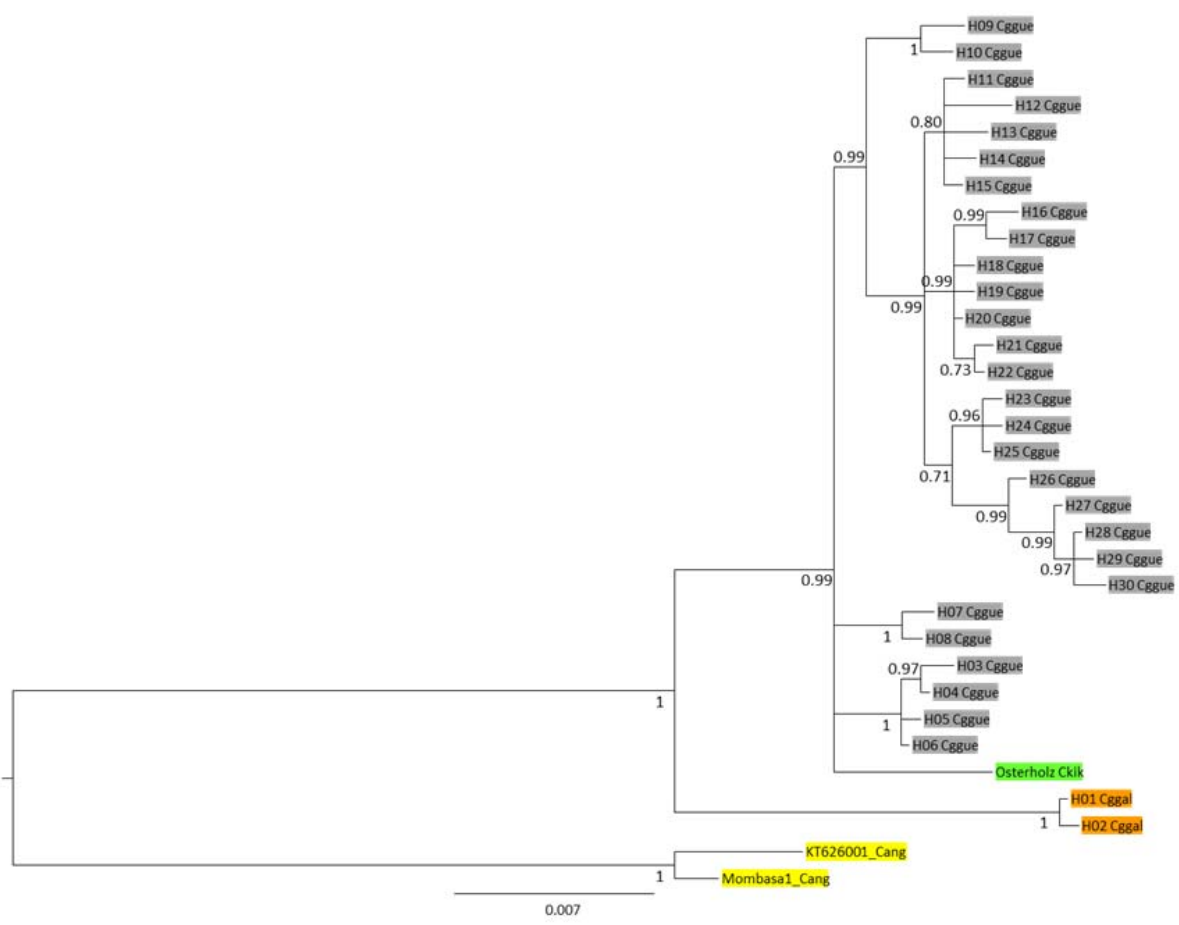

Figure S3c. Bayesian tree (concatenated). Csat $=$ Colobus satanas, Cvel $=$ C. vellerosus, Cpol $=C$. polykomos, Cang $=C$. angolensis, Cgocc $=C$. guereza occidentalis, Cggal $=C . g$. gallarum, Cgmat $=$ C. g. matschiei, Cggue $=$ C. g. guereza, Cgkik =C. g. kikuyuensis, Cgcau $=$ C. g. caudatus, Cgue = C. guereza ssp.. Node labels refer to Bayesian PPs. 
Table S1. Taxonomy, geographical origin (coordinates in decimal degrees) and haplotype assignments of Colobus samples and respective NCBI Accession numbers per haplotype.

\begin{tabular}{|c|c|c|c|c|c|c|c|c|c|c|}
\hline taxon & ID & Country & Site & Lat & Long & NADH hap & Acc Num & cytb hap & Acc Num & comb hap \\
\hline C. satanas & & GNQ & Bioko Island & & & og & EU580054 & & & \\
\hline C. satanas & & GNQ & Bioko Island & & & og & EU580055 & & & \\
\hline C. vellerosus & & GHA & Boabeng-Fiema & & & og & EU580056 & & & \\
\hline C. polykomos & & CIV & Tai National Park & & & og & EU580053 & & & \\
\hline C. angolensis & Mombasa1 & KEN & s of Mombasa & -4.3353 & 39.5689 & og & MK415322 & og1 & MK415348 & og1 \\
\hline C. angolensis & & KEN & & & & & & og1 & HQ859372 & \\
\hline C. angolensis & & & zoo, palliatus? & & & og & EU580047 & & & \\
\hline C. angolensis & & & unknown, zoo & & & og & EU580046 & & & \\
\hline C. angolensis & & & palliatus? & & & og & KT626001 & og2 & KT626001 & og2 \\
\hline C. angolensis & & KEN & & & & & & og2 & HQ859342 & \\
\hline C. g. matschiei? & & & & & & & AY863427 & & & \\
\hline C. g. matschiei & & KEN & Kakamega Forest & & & & EU580051 & & & \\
\hline C. g. matschiei & & & $\mathrm{ZOO} ?$ & & & & DQ399681 & & & \\
\hline C. g. matschiei & & & zoo? & & & & DQ399682 & & & \\
\hline C. g. matschiei & & & zoo? & & & & DQ399685 & & & \\
\hline C. g. matschiei? & & & zoo? & & & & DQ399683 & & & \\
\hline C. g. matschiei? & & & zoo? & & & & DQ399684 & & & \\
\hline C. g. occidentalis & & & zoo? & & & & DQ399697 & & & \\
\hline C. g. occidentalis & & & zoo? & & & & DQ399698 & & & \\
\hline C. g. occidentalis & & CMR & unknown locality & & & & EU580052 & & & \\
\hline C. g. caudatus? & & & zoo, caudatus? & & & & EU580049 & & & \\
\hline C. g. caudatus? & & & zoo? & & & & DQ399690 & & & \\
\hline C. g. caudatus? & & & zoo? & & & & DQ399692 & & & \\
\hline C. g. caudatus? & & & zoo? & & & & DQ399695 & & & \\
\hline C. g. caudatus & & & zoo? & & & & DQ399687 & & & \\
\hline C. g. caudatus & & & zoo? & & & & DQ399688 & & & \\
\hline C. g. caudatus & & & zoo? & & & & DQ399689 & & & \\
\hline C. g. kikuyuensis? & & & zoo, kikuyuensis? & & & & EU580050 & & & \\
\hline C. g. kikuyuensis & & & zoo? & & & & DQ399691 & & & \\
\hline C. g. kikuyuensis & & & zoo? & & & & DQ399694 & & & \\
\hline C. g. kikuyuensis & Osterholz & & zoo, kikuyuensis? & & & & MK415323 & & EU004483 & og \\
\hline C. g. gallarum & ID1 & ETH & Adas Mts Forest & 9.3087 & 41.2449 & h01 & MK415324 & h01 & MK415349 & h01 \\
\hline C. g.gallarum & ID2 & ETH & Adas Mts Forest & 9.3098 & 41.2453 & h01 & & h01 & & h01 \\
\hline
\end{tabular}




\begin{tabular}{|c|c|c|c|c|c|c|c|c|c|c|}
\hline C. g. gallarum & ID3 & ETH & Adas Mts Forest & 9.3106 & 41.2453 & h01 & & h02 & MK415350 & h02 \\
\hline C. g. gallarum & ID4 & ETH & Adas Mts Forest & 9.3106 & 41.2453 & h01 & & h01 & & h01 \\
\hline C. g. gallarum & C1 & ETH & Kuni-Muktart & 9.0208 & 40.8402 & h01 & & h01 & & h01 \\
\hline C. g. gallarum & $\mathrm{C} 2$ & ETH & Kuni-Muktart & 9.0258 & 40.8567 & h01 & & h01 & & h01 \\
\hline C. g. gallarum & $\mathrm{C} 3$ & ETH & Kuni-Muktart & 9.0291 & 40.8424 & h01 & & & & \\
\hline C. g. gallarum & $\mathrm{C} 4$ & ETH & Kuni-Muktart & 9.0292 & 40.8550 & h01 & & h01 & & h01 \\
\hline C. g. gallarum & $\mathrm{C} 5$ & ETH & Kuni-Muktart & 9.0106 & 40.8476 & h01 & & h01 & & h01 \\
\hline C. g. gallarum & C6 & ETH & Kuni-Muktart & 9.0172 & 40.8303 & h01 & & h01 & & h01 \\
\hline C.g.guereza & Ars07 & ETH & Arussi & 7.9853 & 39.6784 & h02 & MK415325 & h03 & MK415351 & h03 \\
\hline C. g. guereza & Ars12 & ETH & Arussi & 7.9853 & 39.6784 & h02 & & h03 & & h03 \\
\hline C. g. guereza & SH17 & ETH & Kama1 & 7.3172 & 36.0702 & h03 & MK415326 & & & \\
\hline C. g. guereza & Ars04 & ETH & Arussi & 7.9853 & 39.6784 & h04 & MK415327 & h05 & MK415353 & h05 \\
\hline C.g.guereza & Bns01 & ETH & Benessa & 6.3121 & 38.5000 & h04 & & h03 & & h04 \\
\hline C. g. guereza & Bns08 & ETH & Benessa & 6.3121 & 38.5000 & h04 & & & & \\
\hline C. g. guereza & SH27 & ETH & Boginda1 & 7.5256 & 36.1180 & h04 & & & & \\
\hline C.g.guereza & SH24 & ETH & Kama1 & 7.3171 & 36.0704 & h04 & & & & \\
\hline C. g. guereza & Wondo26 & ETH & Wondo Genet & 7.0209 & 38.6362 & h04 & & h06 & MK415354 & h06 \\
\hline C. g. guereza & Wondo31 & ETH & Wondo Genet & 7.0209 & 38.6362 & h04 & & h06 & & h06 \\
\hline C. g. guereza & SH31 & ETH & Yayoo & 8.2895 & 35.6091 & h04 & & & & \\
\hline C. g. guereza & SH02 & ETH & Boginda2 & 7.5094 & 36.1302 & h05 & MK415328 & & & \\
\hline C. g. guereza & SH13 & ETH & Boginda2 & 7.5114 & 36.1217 & h05 & & & & \\
\hline C. g. guereza & SH07 & ETH & Kayakela1 & 7.3202 & 36.2224 & h06 & MK415329 & h11 & MK415359 & h07 \\
\hline C. g. guereza & Sha94 & ETH & Shako, Bemch Maji & 6.5000 & 35.0000 & h06 & & h10 & MK415358 & h08 \\
\hline C. g. guereza & SH_ManII & ETH & Managesha II & 8.9668 & 38.5455 & h07 & MK415330 & & & \\
\hline C. g. guereza & SH_ManIII & ETH & Managesha III & 8.9668 & 38.5455 & h07 & & & & \\
\hline C.g.guereza & Bure25 & ETH & Bure/Agew Mider & 11.0180 & 36.7628 & h08 & MK415331 & h18 & MK415366 & h13 \\
\hline C. g. guereza & Har06 & ETH & Harenna & 6.6827 & 39.8366 & h09 & MK415332 & h22 & MK415370 & h29 \\
\hline C. g. guereza & Har07 & ETH & Harenna & 6.6827 & 39.8366 & h09 & & h22 & & h29 \\
\hline C. g. guereza & Yir09 & ETH & Yirgalem & 9.7213 & 37.5724 & h10 & MK415333 & h21 & MK415369 & h30 \\
\hline C. g. guereza & Bns02 & ETH & Benessa & 6.3121 & 38.5000 & h10 & & h22 & & h28 \\
\hline C. g. guereza & Dale02 & ETH & Dale Woreda, Kaffa & 6.4499 & 38.2220 & h10 & & h22 & & h28 \\
\hline C. g. guereza & SH36 & ETH & Harenna 1 & 6.7476 & 39.7139 & h10 & & & & \\
\hline C. g. guereza & Yir12 & ETH & Yirgalem & 9.7213 & 37.5724 & h10 & & h21 & & h30 \\
\hline C. g. guereza & Jma06 & ETH & Jimma & 7.6679 & 36.8861 & h11 & MK415334 & h07 & MK415355 & h09 \\
\hline C. g. guereza & Jma67 & ETH & Jimma & 7.6679 & 36.8861 & h11 & & h07 & & h09 \\
\hline C. g. guereza & Jma91 & ETH & Jimma & 7.6679 & 36.8861 & h11 & & h07 & & h09 \\
\hline C. g. guereza & SH11 & ETH & Boginda2 & 7.5117 & 36.1211 & h12 & MK415335 & h22 & & h27 \\
\hline
\end{tabular}




\begin{tabular}{|c|c|c|c|c|c|c|c|c|c|c|}
\hline C. g. guereza & SH12 & ETH & Boginda2 & 7.5112 & 36.1213 & h12 & & h22 & & h27 \\
\hline C. g. guereza & SH18 & ETH & Kama2 & 7.3193 & 36.0870 & h13 & MK415336 & & & \\
\hline C. g. guereza & SH21 & ETH & Kama1 & 7.3110 & 36.0752 & h14 & MK415337 & & & \\
\hline C. g. guereza & SH08 & ETH & Kayakela2 & 7.3233 & 36.2153 & h15 & MK415338 & & & \\
\hline C. g. guereza & SH03 & ETH & Boginda2 & 7.5128 & 36.1206 & h16 & MK415339 & h26 & MK415374 & h18 \\
\hline C. g. guereza & Bure28 & ETH & Bure/Agew Mider & 11.0180 & 36.7628 & h16 & & h31 & MK415379 & h16 \\
\hline C. g. guereza & Bure32 & ETH & Bure/Agew Mider & 11.0180 & 36.7628 & h16 & & h31 & & h16 \\
\hline C. g. guereza & Bure35 & ETH & Bure/Agew Mider & 11.0180 & 36.7628 & h16 & & h31 & & h16 \\
\hline C. g. guereza & Bure37 & ETH & Bure/Agew Mider & 11.0180 & 36.7628 & h16 & & h31 & & h16 \\
\hline C. g. guereza & Din1a & ETH & Dinibira, Kaffa & 6.1500 & 37.3400 & h16 & & h24 & MK415372 & $\mathrm{h} 20$ \\
\hline C. g. guereza & Din1b & ETH & Dinibira, Kaffa & 6.1500 & 37.3400 & h16 & & h30 & MK415378 & h17 \\
\hline C. g. guereza & SH22 & ETH & Kama1 & 7.3145 & 36.0727 & h16 & & h25 & MK415373 & h19 \\
\hline C. g. guereza & SH25 & ETH & Kayakela2 & 7.3217 & 36.2138 & h16 & & h26 & & h18 \\
\hline C. g. guereza & SH32 & ETH & Yayoo & 8.2895 & 35.6091 & h17 & MK415340 & h29 & MK415377 & $\mathrm{h} 21$ \\
\hline C. g. guereza & Bale01 & ETH & Bale Mts & 7.2200 & 39.7175 & h18 & MK415341 & h24 & & h22 \\
\hline C.g.guereza & Bale02 & ETH & Bale Mts & 7.2200 & 39.7175 & h18 & & h24 & & h22 \\
\hline C. g. guereza & SH04 & ETH & Boginda1 & 7.5170 & 36.1196 & h18 & & h24 & & h22 \\
\hline C.g.guereza & WG06 & ETH & West Gojam & 11.3716 & 37.2656 & h19 & MK415342 & h13 & MK415361 & h15 \\
\hline C. g. guereza & Woch02 & ETH & Wochalle, Wollo & 11.0251 & 39.6839 & h19 & & h17 & MK415365 & h11 \\
\hline C. g. guereza & Woch33 & ETH & Wochalle, Wollo & 11.0251 & 39.6839 & h19 & & h17 & & h11 \\
\hline C.g.guereza & Woch50 & ETH & Wochalle, Wollo & 11.0251 & 39.6839 & h19 & & h17 & & h11 \\
\hline C. g. guereza & SH_WolIIA & ETH & Woliso & 8.5348 & 37.9810 & h19 & & & & \\
\hline C. g. guereza & SH_WolIIB & ETH & Woliso & 8.5348 & 37.9810 & h19 & & & & \\
\hline C. g. guereza & SH33 & ETH & Bale Mts & 7.0443 & 39.5463 & $\mathrm{~h} 20$ & MK415343 & h16 & MK415364 & h12 \\
\hline C. g. guereza & SH34 & ETH & Bale Mts & 7.0443 & 39.5463 & h20 & & h16 & & h12 \\
\hline C. g. guereza & Bed35 & ETH & Bedelle & 8.4592 & 36.3618 & $\mathrm{~h} 21$ & MK415344 & h14 & MK415362 & h14 \\
\hline C. g. guereza & Bed48 & ETH & Bedelle & 8.4592 & 36.3618 & h21 & & h14 & & h14 \\
\hline C. g. guereza & Bure48 & ETH & Bure/Agew Mider & 11.0180 & 36.7628 & h21 & & & & \\
\hline C. g. guereza & Jma01 & ETH & Jimma & 7.6679 & 36.8861 & h21 & & h09 & MK415357 & h10 \\
\hline C. g. guereza & SH05 & ETH & Kama1 & 7.3178 & 36.0695 & $\mathrm{~h} 22$ & MK415345 & h28 & MK415376 & $\mathrm{h} 25$ \\
\hline C.g.guereza & SH10 & ETH & Boginda1 & 7.5331 & 36.1210 & h22 & & h27 & MK415375 & h23 \\
\hline C. g. guereza & SH15 & ETH & Boginda1 & 7.5301 & 36.1208 & h22 & & $\mathrm{h} 27$ & & h23 \\
\hline C. g. guereza & Illi01 & ETH & Illibabour & 8.4879 & 35.6992 & h22 & & h27 & & h26 \\
\hline C. g. guereza & Bed40 & ETH & Bedelle & 8.4592 & 36.3618 & h23 & MK415346 & & & \\
\hline C. g. guereza & SHBab & ETH & Boginda1 & 7.5331 & 36.1210 & h24 & MK415347 & h28 & & h24 \\
\hline C.g.guereza & Ars11 & ETH & Arussi & 7.9853 & 39.6784 & & & h04 & MK415352 & \\
\hline C. g. guereza & Ars09 & ETH & Arussi & 7.9853 & 39.6784 & & & h06 & & \\
\hline
\end{tabular}




\begin{tabular}{|c|c|c|c|c|c|c|c|c|}
\hline C. g. guereza & Wondo32 & ETH & Wondo Genet & 7.0209 & 38.6362 & h06 & & \\
\hline C. g. guereza & SH06 & ETH & Kama2 & 7.3231 & 36.0891 & h07 & & \\
\hline C. g. guereza & Jma02 & ETH & Jimma & 7.6679 & 36.8861 & h08 & MK415356 & \\
\hline C. g. guereza & Gesh02 & ETH & Gesh & 6.3689 & 35.1245 & h12 & MK415360 & \\
\hline C. g. guereza & Bed01 & ETH & Bedelle & 8.4592 & 36.3618 & h15 & MK415363 & \\
\hline C. g. guereza & Woch92 & ETH & Wochalle, Wollo & 11.0251 & 39.6839 & h17 & & \\
\hline C. g. guereza & Har15 & ETH & Harenna & 6.6827 & 39.8366 & h19 & MK415367 & \\
\hline C. g. guereza & Bure47 & ETH & Bure/Agew Mider & 11.0180 & 36.7628 & h20 & MK415368 & \\
\hline C.g.guereza & Dale01 & ETH & Dale Woreda, Kaffa & 6.4499 & 38.2220 & h22 & & \\
\hline C. g. guereza & Har03 & ETH & Harenna & 6.6827 & 39.8366 & h22 & & \\
\hline C. g. guereza & Har04 & ETH & Harenna & 6.6827 & 39.8366 & $\mathrm{~h} 22$ & & \\
\hline C.g.guereza & Har08 & ETH & Harenna & 6.6827 & 39.8366 & $\mathrm{~h} 22$ & & \\
\hline C. g. guereza & Har13 & ETH & Harenna & 6.6827 & 39.8366 & h22 & & \\
\hline C. g. guereza & Har22 & ETH & Harenna & 6.6827 & 39.8366 & $\mathrm{~h} 22$ & & \\
\hline C. g. guereza & Illi02 & ETH & Illibabour & 8.4879 & 35.6992 & h27 & & \\
\hline C.g.guereza & Jma03 & ETH & Jimma & 7.6679 & 36.8861 & h22 & & \\
\hline C. g. guereza & Sha92 & ETH & Shako, Bemch Maji & 6.5000 & 35.0000 & h22 & & \\
\hline C. g. guereza & Sha93 & ETH & Shako, Bemch Maji & 6.5000 & 35.0000 & h23 & MK415371 & \\
\hline
\end{tabular}

CIV = Ivory Coast, CMR = Cameroon, ETH = Ethiopia, GHA = Ghana, GNQ = Equatorial Guinea, KEN = Kenya; $\mathrm{h}=$ haplotype, og = outgroup haplotype 
Table S2. Primers used in this study

\begin{tabular}{|l|l|}
\hline Primer ID & Sequence 5'-3' \\
\hline Cytb1-F & ACC AAT GAT ATG AAA AAY CAT \\
\hline Cytb1-R & TGG TTG TAT AGT AGG GGT \\
\hline Cytb2-F & ACA CCT ACT CTT TCT ACA \\
\hline Cytb2-R & GTT TTG GGT ATT GGY GGT \\
\hline NADH-F & CTA ACC CCT ACG AAT GCG G \\
\hline NADH-R & CGT GAG GGA CTT TCA TTG TG \\
\hline
\end{tabular}

